\title{
Points of Contact Between the Stappian Philosophy and Emergent Aspect Dualism
}

\author{
Christopher W. Tyler $^{1}$
}

Received: 30 January 2019 / Accepted: 25 March 2019 / Published online: 29 April 2019

(C) The Author(s) 2019

\begin{abstract}
Henry Stapp is well known for his advanced views of Quantum Physics and particularly for development of the view that the mind of the observer is intimately involved in the collapse of the wave function when an observation is made. He expands this view to a cosmic mind conceptualization that, in common with much of Quantum Physics, seems to challenge the bounds of rational logic. My own work has been in developing an Emergent Aspect Dualism of the nature of the universe that attempts to build a coherent philosophy of the mental and quantal complexities on an entirely rational basis. Yet, there are numerous points of contact between the two philosophies in the dual aspects of the nature of mind, the interrogative nature of consciousness, the elimination of the physical collapse of the wavefunction, and the creative emergence of the physical complexity of the universe. Where the philosophies diverge is in conceptualizing the existence of a cosmic mind underlying the organization of the physical universe; for Emergent Aspect Dualism the only form of cosmic mind could be the universe itself, operating at a cosmic time scale.
\end{abstract}

Keywords Quantum physics · Probability · Collapse - Wavefunction · Consciousness · Question · Emergence · Creativity · Universe $\cdot$ Mind

A thumbnail sketch of Emergent Aspect Dualism is that the universe as we know is a complex of processes that derive, in common with classical Quantum Physics, from energy as specified by the (continuous form of the) Schrödinger Equation, that processes of successive levels of complexity emerge from this basic energy level (including matter in its various manifestations, as expressed by the Einstein Energy Equation, $E=m c^{2}$ ), that one of those levels of process complexity is what we know as (our) consciousness, that the Hard Problem of consciousness is mainly that "we" are the process that is conscious, and hence this process is the only one that we experience from the "inside" as being that process, thus giving rise to the dual aspects of an inside and an outside view of the processes of consciousness in ourselves and in others, respectively (Tyler 2015). Note that, in assuming that the primordial essence of the universe is energy, this ontology corresponds to an elaboration of the Heraclitan dictum that "all is

Christopher W. Tyler cwtyler2020@gmail.com

1 Optometry and Vision Sciences, City University of London, Northampton Square, London EC1V 0HB, UK flux", as opposed to any of the other underlying substances (or indescribables) proposed by his Ionian counterparts.

Thus, this philosophy is both monistic, in that all derive ultimately from the flow of a single "essence" - energy, and dualistic, in that it recognizes the emergence of an essential dualism between the introspective aspect of the mind and the extrospective aspect of the brain. (It should be emphasized that the levels of "emergence" envisaged here are not a mystical "hard" emergence but a progressive aggregation of the complexity of the monistic substrate such that new, unsuspected principles appear from lower-level simpler ones, as detailed in Tyler 2015.)

What, then, are the mutualities and divergences between the Stappian world view and this philosophy of Emergent Aspect Dualism?

\section{The Dual Aspect Nature of Mind}

In an evident analysis of the Cartesian proposition, "Cogito ergo sum", Stapp says:

Why is consciousness subjective? An actualization even has many components, all of which are integral parts of 
the whole. The totality contains the slowly-changing fringe that constitutes the 'I', or 'psyche', which is felt as the experiencing subject and actualizer. The experiencing subject is part of the thought, not an outside observer of the thought

Stapp (1999, p. 213)

Here, Stapp is implying two distinct aspects of the (conscious) thought process, that of the experiencing subject and that of the outside (objective) observer. Thus, similarly to Emergent Aspect Dualism, Stapp is clearly accepting that thought processes can be experienced either subjectively from the "inside" or objectively from the "outside." Stapp's concern relates to the long-standing issue in philosophy, dating back to Descartes, as to whether the cogito entails the sum a priori, or whether Descartes' formulation that the sum follows logically from the cogito is a valid logical inference. It is certainly the case that the "I" is entailed a priori in the standard translation: "I think, therefore I am." Removing it, the phrase becomes "There is a thought, therefore there is an existence," which does not entail that "I" exist. Making this point, Kierkegaard (1846) points out that a more valid formulation is that existence is already presupposed in order for thinking to occur. These critiques are not, therefore, questioning the basic inference that Descartes' knowledge of his thinking is proof of his existence, just of the formulation of the logical inference.

The most cogent criticism of this kind of formulation is the incidental remark by Lewis Carroll in "Alice in Wonderland" that she is not sure whether she is a figment of the Red King's dream or he of hers. That is, the whole thought process that constitutes the story in the book might not entail her existence but rather the existence of the Red King having a dream about her, raising deep questions about the nature of philosophical investigation itself. If even Descartes' irreducible truth of the nature of our existence can be questioned, there is no firm ground for any conceptualization. Nagel (1974) asked "What is it like to be a bat?"; here, Carroll seems to be asking "What is it like to be a thought?" Is a thought a defined entity that could, in principle, be an independent conscious entity within the mind as a whole?

However, while such outré speculations cannot be dismissed out of hand, they go far beyond the position of Emergent Aspect Dualism, which is that the fundamental essence of existence is energy (or what Heraclitus would call "flux"), and that consciousness is one form of organization that emerges from the underlying energy flux, having the particular property that it can view some aspects of its processes from the "inside," while other processes can view these or other aspects of its processes from the "outside." It is this dual aspect property of such processes that constitutes the Hard
Problem, as was cogently discussed by Bissell (1974). Stapp takes the corresponding emergence to a higher level by treating the physics world as an emergent structure of information.

\section{Interrogative Nature of Consciousness}

A key form of emergence in the Emergent Aspect Dualism is the interrogative nature of consciousness. Tyler (2018) suggested that there is an intimate relationship between consciousness and the ability to formulate a question. A physical system or process cannot ask a question; it can only operate as a process. Computers may come close to it, as in the famous questions asked by the MIT "Eliza" therapist simulation, such as "And how do you feel about your father?" but these are rote formulations without the understanding of what is being asked.

Stapp seems to express a similar view when he talks of the creativity of human free will, the ability to generate its own options rather than simply responding directly to the inputs.

... in the transition to von Neumann quantum theory: the choice of which question will be put to nature, is not controlled by any rules that are known or understood within contemporary physics. This choice associated with a mind-brain-body system is, in this specific sense, a free choice: it is not governed by the physical laws of contemporary physics (i.e., quantum theory). This freedom constitutes a logical "gap" in the dynamical rules of contemporary physical theory.

Stapp (2001, p. 24)

What Stapp seems to be doing here is specifying the role of the observer in the (quantum physical) experiment by drawing a distinction between the simple quantal absorption events that are going on by the gazillions, as the energy streaming through the universe is absorbed by the matter it encounters (which he terms the Dirac Choice), and the deliberate probabilistic situations where a (conscious) observer is setting up a particular situation and asking whether an energy absorption event occurs (which he terms the Heisenberg Choice).

But in the present framework, the important issue is that it is the human observer that formulates the question to be asked of the physical system. The Emergent Aspect Dualism position is that it only makes sense to think of such a scenario as occurring in the thought processes of a conscious mind and that only conscious minds can be said to ask questions and to formulate the potential answers in terms of probabilities (as detailed in Tyler 2018). It is this process of question formulation that Stapp identifies as not being understood by contemporary physics. 


\section{Probabilistic Collapse of the Wavefunction}

According to quantum theory the waveform is associated with an electron produced by radiative decay from a heavy nucleus and will propagate away from the original nucleus in all directions and then suddenly collapse to a small region the size of the water droplets [in a cloud chamber] when the corresponding track in a cloud chamber is observed. This collapse is completely natural for a probability function, and, correspondingly, there is no tendency or propensity for a quantum to be observed in one place immediately after it is observed in a faraway place. [My emphases]

Stapp (2009, p. 96)

Here, Stapp seems to recognize that the nature of the collapse of the wavefunction is an inherent property of the fact that it is a probability function (unlike the continuous energy function of the Schrödinger Equation), and that ultimately a probability function is not something that can exist physically, but must be built up in the mind of the observer (and its mechanistic extensions into electronic systems) by the cumulation over multiple equivalent instances. A probability distribution is a discrete concept that itself has the inherent properties of incorporating the superposition of states, with these complementary states being superposed with probabilities of $p$ and (1-p). Once an observation is made, this current observation being predicted collapses the superposition to one state or the other. These two concepts apply to any form of probability, as in horse racing or weather forecasting, without reference to quantum particles. As a mental construct, therefore, probability per se embodies the key paradoxes of Quantum Physics in any conceptual domain (Tyler 2015). Moreover, in the final sentence, Stapp underlines the consequence that this interpretation eradicates the concept of "the collapse of the wavefunction." (But see the last section for a larger consequence of this view.)

A further aspect of standard Quantum Physics that is questioned (or contested) by Emergent Aspect Dualism (in common with Schrödinger 1952, himself) is the unexplained transition from omnidirectional propagation to the unidirectional particle track, which constitutes a sequence of observations of the condensation of the water droplets along the observed trajectory. If there is no tendency for "it" to be observed in a particular place, or a particular direction in the omnidirectional propagation, the particle track would appear as an undirected random walk rather than a coherent directional trajectory. Only if there is an underlying physical entity constituting some form of particle with defined momentum can the sequence of condensation "collapses" appear as a coherent trajectory. Thus, the reality of the observed trajectories violates the fundamental tenets of Quantum Physics that an observation collapses the wavefunction.

They [particle tracks] certainly do not show us the transformation of a couple of plane waves (representing the colliding particles before the collision) into a number of other plane waves (representing the particles that emerge after the collision).

Schrödinger (1952, p. 240)

Only the Emergent Aspect Dualism account (Tyler 2018) avoids this problem by recognizing that the underlying physical reality has a directional solution to the energy function of the Schrödinger Equation, while the "collapse" is a property of the probabilistic condensation of the vapor into water droplets constituting the discrete observations (independent of human questioning).

\section{Emergence and the Creativity of Human Thought Processes}

Stapp views the (human) brain as housing a generative form of consciousness. "Each creative act brings into existence something fundamentally new: it creates a novel 'emergent' quality." (Stapp 2009, p. 94, Section 4.5.6). Stapp is thus in accord with Emergent Aspect Dualism that the processes of consciousness are emergent in nature. However, for Stapp, the emergence is a freedom from the shackles of prosaic physical causation. The conscious processes of thought gain a causal hold over the physical processes of their neural underpinnings.

Mind is identified with the process or creation. Everything that exists is created by this process, which consists of a well-ordered sequence of creative acts called events. ... Each creative act is a grasping, or prehension, of all that has been created by prior acts in a novel but unified way.

Stapp (2009, p. 93, Section 4.5.1)

And again:

The core idea of Whitehead's thought is, I believe, that the experiential aspects are primary: they control the physical, rather than the other way around. ... The experiential aspects of nature enter into the dynamical rules that determine the unfolding of physical reality by way of needed choices that are specified neither by the deterministic aspects of quantum laws, nor by the random elements that enter into quantum theory. Moreover, these 'free' choices can significantly affect the behaviour of an organism that is associated with a sequence of such free choices. 
Stapp (1998, p. 1)

Thus, Stapp's conceptualization is a hard form of emergence in which not only does consciousness appear from the physical structure of events in the brain, but its resultant conscious events can take control of the causal flow and generate novel aspects that are independent of the causal sequence of the neural substrate (which we take to be governed by the dynamic constraints of the Schrödinger equation).

At this point, Stapp's analysis diverges from Emergent Aspect Dualism, which maintains a full psycho-physical parallelism of the causal flow, such that the causal relations applicable to mental states should be precisely parallel to those of the underlying physical states, which are two aspects of the identical process. Any creativity found in the mental processes (relative to the basic neural connectivity of the information flow) would be equally represented in some way in the neural organization as in the mental constructs. The dualism of Emergent Aspect Dualism is only in the introspective, experiential aspect of the neural events being allowed to seem very different from their external, objective aspects. And indeed, some evident elements of each aspect could be inaccessible for the other aspect, such as the granularity of the specification at the two levels. Some aspects of the neural processing, such as its electrochemical spikes, are inaccessible to the consciousness of the brain it derives from, while some aspects of the conscious processing, such as the qualia, are inaccessible to the recording techniques for objective observation of the neuronal substrate. Nevertheless, for Emergent Aspect Dualism, there is no part of the conscious aspect that is causally independent of the neuronal substrate. As a result, the illusion of creativity may be based on the possibility that the neural processes appear very different from the internal perspective.

While one can sympathize with Stapp's view that the thought processes gain an emergent causative power, because "we" seem to be so much in control of "our" thoughts, it goes against decades of increasingly sophisticated human neuroscience, in which the psychophysiological parallelism is implicitly assumed and is validated with every experiment that measures the brain-level concomitants of thoughts, decisions, judgements, and attentional control operations. With few exceptions, these experiments validate the concept that for every mental state-change, there is a concomitant neural state-change in the brain. Especially in the case of functional imaging studies of the human brain, the emphasis on large-scale patterns of brain organization brings the "grain" of the analysis to a level comparable with the "grain" of human thought processes; reinforcing the view causal relationships at the internally level of conscious thought has parallel causal relationships at the externally accessible level of brain activity. Thus, while recognizing the creative emergence of functional causation at the organizational level of experienced thought processes, Emergent Aspect Dualism posits that the same functional creativity should be identifiable in the objective neural processes, although they may take a very different form of expression. Nevertheless, it is certainly the case that the mind can instigate drastic causal actions against itself, even to the point of eliminating its own existence in the known physical realm by committing suicide. It thus seems difficult to deny some emergent causal role of the mind, at least over the body that nurtures it.

\section{The Universe as a Cosmic Mind}

Over a century ago, the quantum revolution opened the way for cosmic mind. ... Some thinkers point to the incredible fine-tuning of the various constants that must mesh in order for spacetime, matter, and energy to exist: how did this fine-tuning come about? Other thinkers point to the inability of randomness to account for the emergence of DNA and life on Earth. Still others cut the Gordian knot and declare that the human mind is enough to support the existence of cosmic mind - every person is the cosmic mind writ small (or it could be the other way around: universal mind is the human mind writ large).

Chopra and Stapp (2014)

In considering the view that probabilities are restricted to the questioning by some form of mind (see above), Stapp points out that this process could not have taken place prior to the existence of humans or similar life forms, and considers that this argues for the existence of some form of cosmic mind to take this role during the pre-human period. The header quote represents a further expansion of this view to other God-like functions of a cosmic mind. The view that our minds may be a component, or an expression, of an overarching cosmic mind is consistent with ancient Vedic philosophy of the nature of the universe, which has drawn many to make the parallel with the mysterianism of Quantum Physics in extending the domain of observer choice to the ultimate and inaccessible level of quantum events. Indeed, the complexity of the DNA coding, with its multiple levels of coiling structure, its ability to be read out in multiple perfect copies of itself, the cellular ability to perform running repairs on defects in the code, and so on, seems so unimaginably elanorate that many find it difficult to believe that a guiding cosmic mind was not involved in its instantiation.

Emergent Aspect Dualism recognizes the fullness of this complexity, but approaches it from the viewpoint of its physical essence. Thus, this philosophy holds that processes of successive levels of complexity emerge from the basic energy flow that constitutes the universe and that one of those 
emergent levels of process complexity is what we know as our consciousness. In this sense, our consciousness is quite far removed from what are generally considered to be the successive physical, chemical, and biological processes underlying it.

On the other hand, if we focus on the domain of astrophysics, it evidently involves processes of massive complexity constituting suns, solar systems, galaxies, and the large-scale structure of the superclusters. The core concept that consciousness is emergent from processes of sufficient complexity is not in principle limited to biological entities supporting a neural syncytium of sufficient complexity (as it is in many philosophical sources, epitomized by Feinberg and Mallat 2018), but could occur in any dynamic flux-entity that is appropriately structured. Thus, in principle, the astrophysical universe is one such flux-entity and could constitute a cosmic mind per se.

This leads to the question of what is intended by those who conceive of the cosmos as a cosmic mind? The question can be partialled into three levels. One is the mind as a conceptual processing mechanism, a system that can perform operation on conceptual entities conjured up within it and manipulate and transform them into some other (logical) forms, in a process that we call "thinking." Another is as an extroceptive control mechanism that can internalize aspects of structure from outside itself and perform physical operations on them, in a process that we call "behaving." A subset of this second level is the process we call "creating," in which the physical operations of behaving have the effect of transforming the aspects of external structure into more complex entities that themselves may be capable of similar thinking and behaving activities. The third level of conceptualizing of the cosmic mind is as an interactive consciousness that somehow incorporates the consciousnesses of all the sub-elements of this universe of complexity, as though they are its manipulable thought-entities that are at once part of it and yet conceptually separable from the whole. (It should be evident that this description is intended to resonate with the concepts of the universal deity from many world religions, restated in analytic form.)

Given these three levels of conceptualization of the cosmic mind, we can now ask to what extent the known physical universe could be considered to constitute a cosmic mind, or whether its proponents intend an entity beyond or separate from the known physical universe? For the first level, the physical universe involves a host of transformative processes, from the star and galaxy formation to the array of destructive processes that result in supernovae, neutron stars, and black holes. Considered as energy flows, these processes do not appear to be conceptually distinct from the energy flow of the thought processes that we experience in our own minds, so at this level, the universe is sufficiently complex to allow it to operate as a cosmic mind.

The second level of the concept of mind is as an entity that operates on the surroundings outside itself, so invoking the concept of a "mind" tends to bring along this exteroceptive and manipulational aspect of the concept. The physical universe has, by definition, nothing outside it, so it is immediately disqualified from consideration as such a second-level concept of mind. Thus, these familiar representational and externalizational aspects of our own consciousness are not possible to a universal cosmic mind. Moreover, in terms of the creational aspect of this level, the biological facts of our creation as individuals make it evident that our ontology is a self-sufficient process, and the general tenets of the Theory of Evolution are that the entire historical process is similarly selfsufficient and self-sustaining, leaving no place for the involvement of an external creator.

The third level of the cosmic mind concept as a system incorporating conscious subsystems is harder to assess, because it is inherently less well defined by its proponents. At this third level of conceptualization, a mind is taken to have the contents of manipulable thought-entities that could conceivably develop to somehow represent patterns of other thought-entities within it, for example. So, it would be possible to build up a form of self-referential mind that developed and manipulated entities within rather than outside itself. This interactive representation could be the nature of the physical world in some form that we have not, as physicists, yet come to appreciate. Thus, it is recognized that this third level of mind conceptualization is one that could, in principle, operate as a form of cosmic (representational) mind that describes the universe as we understand it.

However, there is a key impediment to accepting the view of the physical universe as a cosmic mind as a meaningful description, and that is the limiting fact of the velocity of light. On the cosmic scale of the physical universe, communication is ponderously slow because its elements are so far apart. If the communications among the stars and galaxies of the universe form some kind of cosmic mind, it is one in which a single 'thought' takes many human lifetimes. Due to this timing discrepancy, there is no possibility that our minds, with their thought processes in the millisecond range, could be a subcomponent of such a universal physical consciousness, with its communication delays spanning eons of time.

Thus, any cosmic mind of which we are a component would have to be a previously unencountered cosmic entity rather than any aspect of the known universe, which may correspond to Stapp's view. Moreover, it would have to have a speed of communication far beyond the Einsteinian limit of the velocity of light in order to reach across the cosmos at communication rates comparable with our individual minds. One may suppose that the cosmic mind enthusiasts might pin their hopes on the recent astrophysical analyses suggesting that the universe contains an undiscovered quotient of dark energy, but it would have to be a novel form of energy propagating at massively superluminal speeds in order to overcome the distance barrier to meaningful complexity at the cosmic scale. And the 
theoretical calculations on this issue change so rapidly that they make it difficult to consider that the existence of dark energy per se is a realistic supposition (see Ali \& Das 2015).

In summary, if there is a cosmic mind with any form of connection to the mental dynamics of our own individual consciousness, it could not have a form compatible with the structures of the known relativistic universe. Returning to the issue of the rationale for the cosmic mind, it is the position of Emergent Aspect Dualism that the quantum probability conundrum arises only in systems being contemplated by a questioning (human) mind. It is considered an overgeneralization to postulate that every energetic absorption in the universe is subject to a mental representation of its probabilities. Instead, it is assumed that they proceed according to the dictates of the continuous Schrödinger Equation (see Rashkovskiy 2016, for a formal derivation of this approach), avoiding the need to postulate a cosmic mind to account for pre-human physics.

\section{Conclusion}

This brief overview finds much in common between Stapp's philosophy and Emergent Aspect Dualism, particularly in the role of the conscious observer in defining the nature of the experimental situation. But there is ultimately a divergence between Stapp's cosmic quantal interpretation into an unsupportable conceptualization of the role of a cosmic mind in local consciousnesses and the elaborated monism of the Emergent Aspect Dualism which, despite its name, is at heart a fundamental realism based on the concept that, "all is flux," though heavily disguised as elaborated forms of matter, quantization, and consciousness.

Acknowledgments Thanks to Stan Klein for extensive discussions about the fundamentals of Quantum Physics over the years and for the invitation to participate in the present festschrift for Henry Stapp.

\section{Compliance with Ethical Standards}

Conflict of Interest The author declares that he has no conflict of interest.
Open Access This article is distributed under the terms of the Creative Commons Attribution 4.0 International License (http:// creativecommons.org/licenses/by/4.0/), which permits unrestricted use, distribution, and reproduction in any medium, provided you give appropriate credit to the original author(s) and the source, provide a link to the Creative Commons license, and indicate if changes were made.

\section{References}

Ali, A. F., \& Das, S. (2015) Cosmologyfrom quantum potencial. Physics Letter B, 741, 276-279.

Bissell, R. E. (1974). A dual-aspect approach to the mind-body problem. Reason Papers., 1, 18-39.

Chopra, D., \& Stapp, H. P. (2014). Is a mind-element needed to interpret quantum mechanics? Do physically undetermined choices enter into the evolution of the physical universe? The Blog, Huffington Post, 9/13/2014

Feinberg, T. E., \& Mallat, J. M. (2018). Consciousness Demystified. Cambridge: MIT Press.

Kierkegaard, S. (1846, 1985). Concluding unscientific postscript. In Philosophical Fragments (p. 38-42). Trans. Hong, Princeton.

Nagel, T. (1974). What is it like to be a bat? Philosophical Review, 83, 435-456.

Rashkovskiy, S. A. (2016) Classical-field description of the quantum effects in the light-atom interaction. arXiv preprint arXiv: 1603.02102.

Schrödinger, E. (1952). Are there quantum jumps? Part II. British Journal for the Philosophy of Science, 3, 233-242.

Stapp, H. P. (1998). Whiteheadian process and quantum theory of mind. Lawrence Berkeley National Laboratory Report, LBNL-42143.

Stapp, H. P. (1999). Attention, intention, and will in quantum physics. Lawrence Berkeley National Laboratory Report, LBNL-42650.

Stapp, H. P. (2001). Quantum theory and the role of mind in nature. Lawrence Berkeley National Laboratory Report, LBNL-44712. arXiv:quant-ph/0103043.

Stapp, H. P. (2009). The role of human beings in the quantum universe. World Futures, 65, 7-18.

Tyler, C. W. (2015). The emergent dualism view of quantum physics and consciousness. Cosmos and History, 11, 97-114.

Tyler, C. W. (2018). The emergent aspect dualism view of quantum physics: a new ontology to resolve the complementarity conundrum. Journal of Research in Philosophy and History, 1, 166-182. https://doi.org/10.22158/jrph.v1n2p166.

Publisher's Note Springer Nature remains neutral with regard to jurisdictional claims in published maps and institutional affiliations. 